\title{
On the collection of photocurrent in solar cells with a contact grid
}

\author{
A.V. Sachenko, A.P. Gorban' \\ Institute of Semiconductor Physics of NASU,45, prospect Nauki, 252028 Kiev, Ukraine
}

\begin{abstract}
The exact solution for the dependence of specific power of photoconversion in the mode of maximum collected power on distance $l$ between lines of the contact grid has been obtained. It is shown that in an optimized case, when the change of the potential of heavily doped front layer under contacts and between contacts is less than $k T / q$, the characteristic length $L$ can be introduced with a meaning of the distance at which the photocurrent reduces by a factor of $e$ due to recombination. Variation of the filling factor of SC IVC due to the presence of contact grid is then analytically expressed via this length.

It is found that in unoptimized case, when the distance between contact strips $l$ is much longer than $L$, the photocurrent collection is determined by lesser, as compared to $L$, distance, at which the front layer potential changes from the value of $V_{m}$ under contacts to the open- circuit voltage between the contacts. In this case the change of IVC filling factor due to the presence of contact grid is expressed again analytically via this new characteristic length.

In the intermediate case, when $l \approx L$, the solution of the problem can be found by numerical methods only.
\end{abstract}

Keywords: photocurrent collection, contact grid, specific power of photoconversion

Paper received 12.05.99; revised manuscript received 06.07.99; accepted for publication 12.07.99.

\section{Introduction}

While considering the question about Ohmic power losses in solar cells (SC) with contact grid the idea is commonly used of sheet resistance of the thin front layer in which the collected current flows and which often provides a main contribution into the series resistance of SC $R_{S}$ [1-4]. In papers [5-7] the notion of photocurrent collection length $L$ in the front layer was introduced, with a physical meaning of a distance at which the photocurrent reduces by a factor of $e$ due to recombination. The length $L$ allows to estimate rather easily the power losses in SC with contact grid composed of the set of parallel metallic strips.

It should be noted that the very notion of series resistance of SC $R_{S}$ is valid, in a strict sense, only for an optimized structure, where the variation of potential along the front layer between contacts is small as compared to $k T / q$ (or, which is the same, the distance between contacts $l$ is less than $L$ ). In the case when the mentioned modulation is comparable to $k T / q$, (and $l \geq 2 L$ ), the use of the notions of series resistance in calculations of Ohmic power losses in $\mathrm{SC}$ becomes, generally, impossible, and the magnitude and the physical sense of the effective photocurrent collection length change.
In this paper the filling factor of SC I-V characteristics is calculated for a general case when the relation between $l$ and $L$ is arbitrary. It is shown that the use of the notion of the effective photocurrent collection length allows to simplify essentially the calculation of the filling factor of IVC and to generalize it for the case of an arbitrary number of recombination channels.

\section{Formulation of the problem}

Let us calculate the specific power of photoconversion for the symmetrical structure shown in Figure 1. We assume that the photocurrent flowing in the front $n^{+}$- layer in the $z$ direction can be described in frames of the one-exponential model of IVC:

$J(z)=J_{s c}-J_{0} \cdot(\exp (u(z) / A)-1)$,

where $J(z)$ is the photocurrent density, $J_{s c}$ is the density of short-circuit current, $J_{0}$ is the density of dark current, $u(z)=q V(z) / k T$ is the dimensionless potential of front $n^{+}-$region, $A$ is the ideality factor of IVC. In the same way as in [6], we consider the linear case in assumption that $\Delta N \ll N_{0}$, where $\Delta N$ and $N_{0}$ are integrated over the 


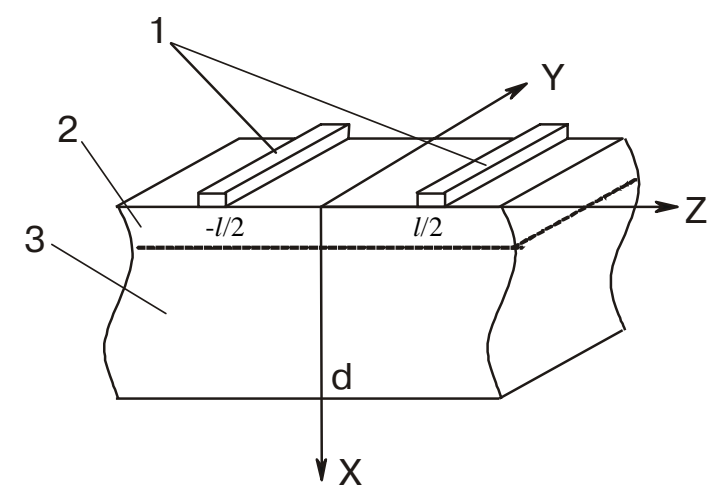

Fig.1. The layout of the structure of the solar cell with contact grid: $1-$ metal contacts, $2-n^{+}$- region, 3 - quasi-neutral bulk of $p$-type.

thickness SC concentrations of extra and equilibrium electrons. Then the continuity equation for the photocurrent flowing in the region $-l / 2<z<l / 2$ according to [6] has the following form

$k T \mu_{n} N_{0} \cdot \frac{d^{2} u(z)}{d z^{2}}-J(z)=0$,

where $\mu_{n}$ is the mobility of electrons in $n^{+}-$layer.

In virtue of the symmetry of the structure, it is sufficient to solve Eq. (2) in the range $0<z<l / 2$, with the following boundary conditions:

$J(l / 2)=J_{m}=J_{s c}-J_{0}\left(\exp \left(\frac{u_{m}}{A}\right)-1\right)$

$\left(\frac{d u(z)}{d z}\right)_{z=0}=0$

where $u_{m}=u_{o c}-A \cdot \ln \left(\frac{u_{o c}}{A}\right)$ is dimensionless voltage of $\mathrm{SC}$ in the mode of maximum power collected, $u_{o c}=A \cdot \ln \left(\frac{J_{S c}}{J_{0}}\right)$ is the dimensionless voltage of open circuit.

The magnitude of photoconversion power for SC of unit area is determined as

$$
W=\frac{2}{l} \int_{0}^{l / 2} W(z) d z=\frac{2}{l} \cdot \frac{k T}{q} \int_{0}^{l / 2} J(z) u(z) d z,
$$

and the filling factor of IVC, $K$, is equal to:

$$
K=\frac{W}{J_{s c} \cdot V_{o c}}
$$

where $V_{o c}=\frac{k T}{q} u_{o c}$ is the open-circuit voltage.

The continuity equation for the electron current flowing in the $n^{+}-$region is the analog of the Poisson equation and can be solved in the similar way. Making transition from the integration on coordinate $z$ to integration on $u(z)$ we get the first integral, and the second integral is taken in quadratures. Then, using the boundary conditions (3) and (4) we get the equation connecting $u$ and $z$ :

$$
\begin{aligned}
& \int_{u(0)}^{u(z)} \frac{d u}{\left((u(0)-u(z))+\frac{A \cdot J_{0}}{J_{s c}}\left(\exp \left(\frac{u(z)}{A}\right)-\exp \left(\frac{u(0)}{A}\right)\right)\right)^{1 / 2}}= \\
& =-\frac{z}{L_{1}}
\end{aligned}
$$

where $L_{1}=\left(\frac{k T \mu_{n} \cdot N_{0}}{2 J_{s c}}\right)^{1 / 2}$ is the characteristic length.

The constant of integration $u(0)$ is found from the equation

$$
\begin{aligned}
& \int_{u(0)}^{u_{m}} \frac{d u}{\left((u(0)-u(z))+\frac{A \cdot J_{0}}{J_{S c}}\left(\exp \left(\frac{u(z)}{A}\right)-\exp \left(\frac{u(0)}{A}\right)\right)\right)^{1 / 2}}= \\
& =-\frac{l}{2 \cdot L_{1}}
\end{aligned}
$$

The equation set (5)-(8) completely determines the IVC filling factor $K$, however, in a general case, it can be solved only numerically. Let us denote

$K=K_{1} \cdot K_{2}$,

where $K_{1}=\frac{J_{m} \cdot V_{m}}{J_{s c} \cdot V_{o c}}$,

$K_{2}=\frac{W}{J_{m} \cdot V_{m}}$

and $W$ is determined by the formula (5). The magnitude $K_{2}$ determined in this way reflects only the influence of the contact grid on the IVC filling factor.

In the case when the separation between the strips of the contact grid is sufficiently small, so that modulation of $V(z)$ is less than $k T / q$, equation (2) is reduced to the diffusion equation with the length

$$
L_{2}=\left(\frac{q \mu_{n} \cdot N_{0} \cdot V_{o c}}{A \cdot J_{s c}}\right)^{1 / 2}
$$

and $K_{2}$ is determined in this case as

$$
K_{2}=\frac{2 L_{2}}{l} \cdot \tanh \left(\frac{l}{2 L_{2}}\right)
$$

As the analysis shows, for the considered case the generalization of the expression for $L_{2}$ can be performed without using the IVC of the form (1). At an arbitrary relation between contributions of different recombination channels into the total recombination current the following equation is valid: 
$L_{2}=\left(\frac{\mu_{n} \cdot N_{0} \cdot k T}{J_{s c}-J_{m}}\right)^{1 / 2}$

The difference $J_{s c}-J_{m}$ is the density of the total recombination current in the mode of maximum power collected. The relation (13) is valid, in particular, at the nonlinear level of excitation in the quasi-neutral region of SC.

\section{Discussion of the results}

In Fig. 2 shown are the dependencies of $K_{2}$ on the distance between strips of the contact grid $l$. The next parameters were used for calculations: $p_{0}=10^{16} \mathrm{~cm}^{-3}, J_{S C}=$ $0.04 \mathrm{~A} / \mathrm{cm}^{2}, J_{0}=3.210^{-12} \mathrm{~A} / \mathrm{cm}^{2}, A=1, N_{0}=10^{15} \mathrm{~cm}^{-2}$, $\mu_{n}=10^{2} \mathrm{~cm}^{2} / \mathrm{V} \mathrm{s}$. The curve 1 is the exact one, it is obtained by the numerical integration of Eqs (5), (7), (8). The curve 2 is plotted according to Eqs (11), (12) for the value of $L_{2}$ equal, at the mentioned parameters, to $0.491 \mathrm{~cm}$. As can be seen from the figure, at $l / L_{2} \leq 1$ the curve 2 agrees well with the exact dependence. The curve 3 is obtained from the approximate solution of equation (7) consisting in neglecting the second addend in its denominator. As a result, the quadratic dependence of $V(z)$ on coordinate $z$ is obtained, which is commonly used in calculations of the photoconversion power losses in frames of an approach with the series resistance [4]. As can be seen, the dependence described by the curve (3) gives incorrect result at $l / L_{2} \geq 1$ (including the possible change of sign). The curve (4) is plotted according to the equation of the form (12) with the effective photocurrent collection length $L_{3}$ equal to $0.241 \mathrm{~cm}$ and obtained from the asymptotic of the $K_{2}(l)$ exact dependence at $l \gg L_{2}$. Within an accuracy of about $5 \% L_{3}$ coincides with the value $L_{4}=2 L_{1} \sqrt{A \cdot \ln \left(u_{o c} / A\right)}=0.255 \mathrm{~cm}$. The magnitude $L_{4}$ is equal to the distance, at which potential $V(z)$ in the unoptimized structure, obtained in the above approximation, varies from the value of $V_{m}$ at the contact to the value of $V_{o c}$ between the contacts. In this case the current is collected only within distances from the contacts that are less than $L_{4}$, whereas at longer distances the open

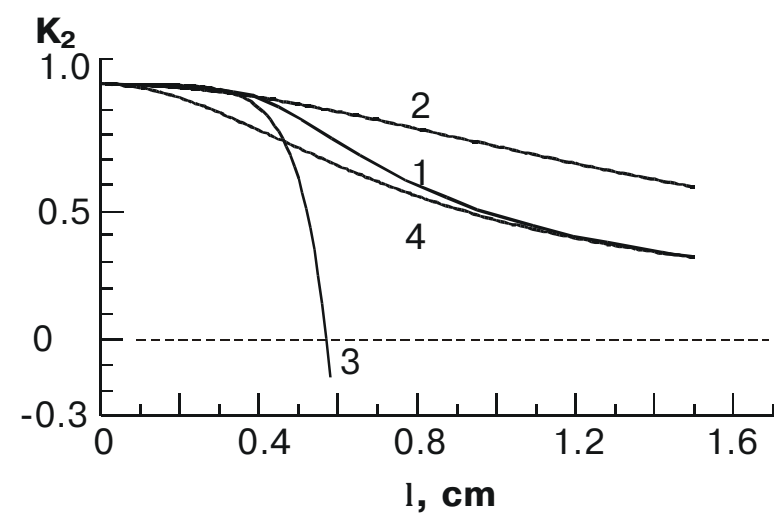

Fig.2. Dependence of variation of the filling factor of IVC related to the presence of the contact grid on the distance between the contacts. circuit mode is realized, and the flowing current is equal to zero. It should be noted that in spite of strong nonlinearity of the expression for $W(z)$ in the considered case, the variation of the filling factor of IVC $K_{2}$ related to the presence of the contact grid can be described by the equation of the form (12) beginning from the values $l>2 L_{2}$.

\section{Conclusions}

In the present paper the theoretical approach is developed which allow to describe exactly the dependence of the IVC filling factor for SC on the distance between the lines of the contact grid $l$ at arbitrary values of $l$. It has been shown that in limiting cases of short and long distances between the contacts in comparison with the photocurrent collection length, the simple equation of the form (12) is valid for the dependence $K_{2}(l)$ with the length $L_{2}$ when $l / L_{2} \leq 1$, and the length $L_{3}$ at $l / L_{2} \geq 2$.

\section{References}

1. R.J. Handy. Theoretical analysis of the series resistance of solar cells/ / Solid -St. Electron .10(8), pp.765-775 (1967)

2. N.C. Wyeth. Sheet resistance component of series resistance in a solar cell as a function of grid geometry// Solid -St. Electron .20(7), pp.629-634 ( 1977)

3. A.M.Vasil'ev, A.P.Landsman. Poluprovodnikovye fotopreobrazovateli (Semiconductors Photoconvertors). Moscow: Sovetskoe Radio, 1971 (in Russian).

4. A.L.Fahrenbruch, R.H.Bube. Fundamentals of solar cells. Photovoltaic solar energy conversion, New York, 1983

5. A.V.Sachenko, A.I.Shkrebtii. Mechanizmy sobiraniya fototoka v solnechnykh elementakh s kontaktnoi setkoi (Mechanisms of photocurrent collection in solar cells with contact grid) // Ukr. Fiz. J., 29 (12), pp.1855-1859 (1984) (in Russian).

6. A.V. Sachenko, A.I.Shkrebtii, T.V.Panichevskaya. Teoreticheskaya model solnechnogo elementa s kontaktnoy setkoy (Theoretical model of solar cell with contact grid) // Optoelectronika i Poluprovodnikovaya Tekhnika, No.21, pp.63-70 ( 1991) (in Russian).

7. A.P.Gorban', A.V. Sachenko,S.I.Frolov. Analiz effektivnosti kremnievykh fotopreobrazovatelei s indutsirovannymi bar'erami $\mathrm{v}$ usloviyakh var'iruemogo spectral'nogo sostava solnechnogo izlucheniya (The analysis of efficiency of silicon photoconvertors with induced barriers in conditions of variable spectral composition of solar radiation) // Optoelectronika i Poluprovodnikovaya Tekhnika, No.16, pp.80-84 (1989) (in Russian). 